The Bangladesh Veterinarian (2010) 27(2) : 56 - 61

\title{
A controlled study to assess the effects of vaccination against Newcastle disease in village chickens
}

\author{
L. R. Barman ${ }^{*}$, M. F. Flensburg2 ${ }^{2}$ A. Permin², M. Madsen 3 and M. R. Islam \\ Department of Pathology, Faculty of Veterinary Science, Bangladesh Agricultural \\ University, Mymensingh-2202, Bangladesh
}

\begin{abstract}
The effect of vaccination against Newcastle disease (ND) in free range village chickens was assessed in a controlled field study. Based on the results of a baseline survey, 12 vaccinated and 12 unvaccinated backyard chicken flocks from two adjacent villages were selected and followed for six months. Serum antibody levels were measured on four occasions. All mortalities were recorded and the cause of death was established by necropsy. Selected ND-suspected cases were subjected to virus isolation, and pathogenicity of two selected isolates was measured in terms of mean death time (MDT) of inoculated chicken embryos. The baseline survey revealed that approximately $90 \%$ of the village families reared chickens of which only $11 \%$ regularly vaccinated their chickens against ND. Another 14\% occasionally vaccinated their chickens and $75 \%$ did not vaccinate at all. In the flocks under study, antibody titres increased in vaccinated and unvaccinated chickens. About $75 \%$ of unvaccinated and $96 \%$ of vaccinated mature chickens (>28 weeks of age) had antibodies to ND virus (NDV), but the level was significantly higher in vaccinated birds. A striking difference in seroconversion was observed in unvaccinated and vaccinated younger birds: $3.3 \%$ vs. $55.1 \%$ in chicks ( $<9$ weeks of age) and $7.3 \%$ vs. $80.3 \%$ in growing pullets (9-28 weeks of age), respectively. Mortality due to ND was significantly higher in unvaccinated flocks (21.6\%) than in vaccinated flocks (4.9\%). Most of these mortalities occurred in growing pullets and chicks. Eight selected samples were positive for NDV when subjected to embryo inoculation and haemagglutination inhibition (HI) test. Two of the isolates were further characterized: both were velogenic pathotype with MDT of $<60$ hours. It is suggested that vaccination against ND could significantly reduce mortality in village chickens. (Bangl. vet. 2010. Vol. 27, No. 2, 56 - 61)
\end{abstract}

\section{Introduction}

Newcastle disease is considered to be one of the most important viral diseases in chickens throughout the world and has a devastating effect on poultry production in most countries (Alexander, 2001). Almost every rural community in Bangladesh keeps small flocks of indigenous chickens (Aini, 1990). The majority of farmers keep their chickens in a scavenging system, where the main source of feed is household

1 Present address: Mirpur Central Poultry Farm, Department of Livestock Services, Dhaka, Bangladesh

2 The Royal Veterinary and Agricultural University, DK-1870 Frederiksberg C., Denmark

3 Danish Veterinary Institute, Hangøvej 2, 8200 Aarhus N, Denmark

* Corresponding author. E-mail: lalitalalita2002@hotmail.com 
refuse and picking from the surroundings. Before the advent of avian influenza, ND was considered as the cause of highest economic loss in village chickens in most developing countries including Bangladesh (Chowdhury et al., 1982; Alexander, 2001). Data on the epidemiology of ND and the impact of vaccination against ND in backyard production systems is limited (Otim et al., 2007; Harrison and Alders, 2010).

The aim of the present study was to assess the impact of vaccination against ND in village chickens through questionnaire survey and a controlled study including seroprofiling, clinical and post-mortem observations, and virus isolation, identification and characterization.

\section{Materials and Methods}

\section{Study area and population}

Two villages, Mujati and Char Adhpakhia, were randomly selected from Muktagacha upazilla in the district of Mymensingh, Bangladesh. The chickens were considered as one population due to the close contact between the villages. Based on the baseline survey data the farmers were divided into three categories: Category 1 farmers applied Baby Chick Ranikhet Disease Vaccine (BCRDV) (F-strain) and Ranikhet Disease Vaccine (RDV) (M-strain) on a routine basis; Category 2 farmers used BCRDV and RDV vaccines occasionally and Category 3 farmers used no vaccine against ND. Selected farmers from Category 1 and 3 with more than 10 chickens were asked to participate in the present study. From Category 1 six flocks were selected randomly in each of the two villages. To select Category three farmers, each village was first divided into six areas. Within each area, one flock was chosen randomly. In total 12 vaccinated and 12 unvaccinated flocks were selected. No birds were allowed to enter the flocks after selection. Birds were considered as chicks when below nine weeks of age, growing pullets at nine to 28 weeks and mature birds above 28 weeks. All birds in the flocks were marked with individual numbers on tags attached to the legs.

\section{Collection and examination of serum samples}

Blood was collected from all flocks on four occasions at one and half month intervals. The samples were left for 2-3 hours in order to separate serum. The serum samples were transferred to Eppendorf tubes, marked with the date and tag number and stored at $-20^{\circ} \mathrm{C}$. The samples were examined using the $\mathrm{HI}$ test to measure antibodies (Anon. 1996). Haemagglutinating (HA) antigen for HI test was prepared in the laboratory as allantoic fluid harvested from embryonated chicken eggs inoculated with F strain of NDV following the standard procedure (Anon. 1996).

\section{Mortality records}

During the study period, the village farmers were requested to submit all dead birds to the investigator. It was emphasized that farmers should not sell or eat their chickens. To ensure that all dead birds were examined, compensation was given. 
During the post-mortem examinations ND-suspected samples were collected and stored at $-20^{\circ} \mathrm{C}$ for further investigations (see below).

Virus isolation, identification and characterization

From selected ND-suspected samples, affected parts of the lungs, spleens, livers, trachea and brains were stored in $50 \%$ buffered glycerol in sterile screw-capped glass bottles marked with sample date, location, age, sex, bird number, history and clinical signs. All samples were kept at $-20^{\circ} \mathrm{C}$. Tissue specimens from each bird were pooled and homogenized to make $20 \%$ suspension in phosphate buffered saline (PBS) containing penicillin $(1000 \mathrm{iu} / \mathrm{ml})$ and streptomycin $(1 \mathrm{mg} / \mathrm{ml})$. The suspension was clarified by low-speed centrifugation and inoculated in embryonated chicken eggs through allantoic route to isolate the virus as described by Hitchner et al. (1975) and Anon. (1996). After death of the embryos the allantoic fluid was harvested, tested for HA activities and subjected to HI test with known anti-NDV hyperimmune serum. Pathogenicity of selected isolates was evaluated by observing mean death time (MDT) of inoculated embryos (Hitchner et al., 1975). All eggs used in the process of NDV isolation, identification, and characterization were obtained from the poultry farm at the Bangladesh Agricultural University.

\section{Statistical analyses}

A chi-square test was used to compare the mortality in unvaccinated and vaccinated birds and in different age and sex groups. To measure the differences in the antibody level in unvaccinated and vaccinated birds an analysis of variance was used. All hypotheses were tested at a 5\% significance level.

\section{Results and Discussion}

Baseline data and the profile of flocks under study

The baseline survey revealed that Mujati and Char Adhapkhia villages had in total 1,380 families, of which 1,225 reared chickens (89\%). All chickens were Desi (indigenous). In total, the two villages comprised around 16,000 chickens - the flock size was on average 13 birds per family. Only $11.1 \%$ of the farmers vaccinated their chickens regularly, $13.9 \%$ occasionally and $75.0 \%$ never. The age and sex distribution of chickens in the 12 vaccinated and 12 unvaccinated flocks is given in Table 1.

\section{Serological investigation}

At the beginning of the study $75.0 \%$ of the mature birds, $7.3 \%$ of the growing pullets and $3.3 \%$ of the chicks in unvaccinated flocks carried antibodies against NDV. In vaccinated flocks, $96.2 \%$ of the mature birds, $80.3 \%$ of the growing pullets and $55.1 \%$ of the chicks had antibodies. The relatively high seropositivity in unvaccinated birds would indicate a constant natural exposure to NDV in village chickens. This result is in agreement with Bell and Moulodi (1988), who found 5-83\% (average 35\%) seropositive village chickens in Morocco. Agebede et al. (1992) found an average of $47 \%$ seropositive village chickens in Cameroon. 
Table 1. Age and sex distribution of chickens in the vaccinated and unvaccinated flocks selected for the study

\begin{tabular}{l|c|c|c|c|c|c}
\hline \multirow{2}{*}{$\begin{array}{c}\text { Vaccination } \\
\text { status }\end{array}$} & \multicolumn{6}{|c}{ Number (\%) of chickens } \\
\cline { 2 - 7 } & Total & $<9$ weeks & 9 to 28 weeks & $>28$ weeks & Male & Female \\
\hline Unvaccinated & 241 & $88(36.5 \%)$ & $\begin{array}{c}102 \\
(42.3 \%)\end{array}$ & $\begin{array}{c}51 \\
(21.2 \%)\end{array}$ & $60(24.9 \%)$ & $181(75.1 \%)$ \\
\hline Vaccinated & 206 & $69(33.5 \%)$ & $\begin{array}{c}83 \\
(40.3 \%)\end{array}$ & $\begin{array}{c}54 \\
(26.2 \%)\end{array}$ & $50(24.3 \%)$ & $156(75.7 \%)$ \\
& & & 185 & 105 & 110 & 337 \\
\hline Total & 447 & 157 & \multicolumn{5}{c}{} \\
\hline
\end{tabular}

An overview of the antibody level in unvaccinated and vaccinated birds is presented in Fig. 1. The level of antibody increased with age in unvaccinated and vaccinated chickens and there was a highly significant $(\mathrm{P}<0.01)$ difference in the NDV antibody level between the different age groups. This finding supports Thitissak et al. (1989) who observed that mean HI titres rose steadily as the birds aged, peaking in unvaccinated birds at 3 years of age. Schmidt and Schmidt (1955) considered HI titre 32 and above to be protective against NDV. Most of the older birds had a protective level of antibodies even without vaccination. However, a large number of unvaccinated growing pullets, particularly during the first three sampling occasions, remained unprotected. On all three occasions a significant difference in the mean antibody level was found between vaccinated and unvaccinated flocks $(\mathrm{P}<0.01)$ in all three age groups.

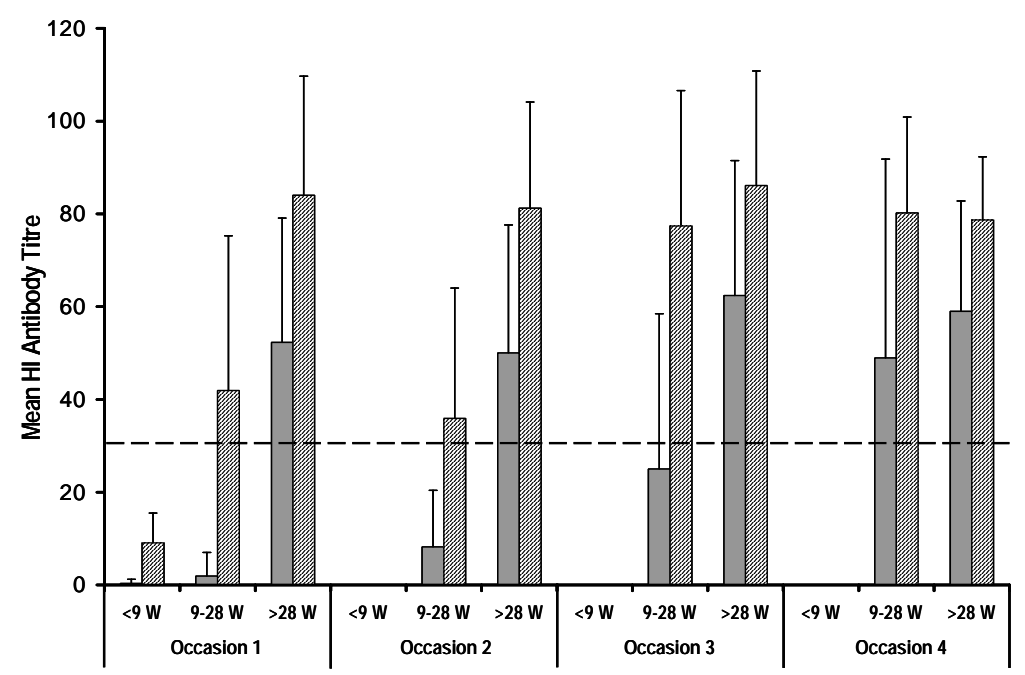

Fig. 1. Mean HI antibody titre against NDV in unvaccinated (solid bar) and vaccinated (striped bar) chickens on four different occasions. Vertical limits indicate standard deviations and the horizontal broken line represents protective level of antibody titre. No chicks ( $<9$ weeks) were available for sampling after the first occasion 


\section{Mortality patterns}

Mortality due to ND was significantly higher $(\mathrm{P}<0.01)$ in the unvaccinated birds (Table 2). Out of 241 unvaccinated birds 52 (21.6\%) died due to ND, whereas out of 206 vaccinated birds, $10(4.9 \%)$ died of ND. The risk of dying from ND was 4.4 times higher in unvaccinated birds. In vaccinated flocks the deaths from ND might be due to improper vaccination (failure to maintain cool chain or improper timing). Diseases other than ND that accounted for mortality included parasitic infections, fowl cholera, chronic respiratory disease, fowl pox, pullorum disease and colibacillosis. The total mortality was significantly $(\mathrm{P}<0.01)$ higher in unvaccinated $(58.1 \%)$ than in vaccinated birds (39.8\%). The risk was 1.5 times higher in unvaccinated birds.

The contribution of ND to overall mortality in unvaccinated and vaccinated chickens of different age and sex groups can be seen in Table 3. In unvaccinated birds mortality due to ND was significantly $(\mathrm{P}<0.01)$ higher in growing pullets than in chicks and mature birds. This result is in agreement with Ezeokoli et al. (1984), who suggested that in backyard management systems birds around 16-24 weeks of age had the highest risk of NDV infection. Differences in mortality due to ND between male and female birds were not statistically significant.

Table 2. Mortality in unvaccinated and vaccinated flocks

\begin{tabular}{l|c|c|c|c|c}
\hline $\begin{array}{c}\text { Vaccination } \\
\text { status }\end{array}$ & $\begin{array}{c}\text { Total } \\
\text { birds }\end{array}$ & $\begin{array}{c}\text { Total birds } \\
\text { lost }\end{array}$ & $\begin{array}{c}\text { Death due } \\
\text { to ND }\end{array}$ & $\begin{array}{c}\text { Death due to } \\
\text { other diseases }\end{array}$ & $\begin{array}{c}\text { Lost due to predators or } \\
\text { simple disappearance }\end{array}$ \\
\hline Unvaccinated & 241 & $140(58.1 \%)^{*}$ & $52(21.6 \%)^{*}$ & $45(18.7 \%)$ & $43(17.8 \%)$ \\
Vaccinated & 206 & $82(39.8 \%)$ & $10(4.9 \%)$ & $38(18.4 \%)$ & $34(16.5 \%)$ \\
Relative risk & & 1.5 & 4.4 & - & - \\
\hline
\end{tabular}

* Significantly higher $(\mathrm{P}<0.01)$

Table 3. Contribution of ND to overall mortality in different age and sex groups of unvaccinated and vaccinated chickens

\begin{tabular}{c|c|c|c|c|c}
\hline \multirow{2}{*}{$\begin{array}{c}\text { Vaccination } \\
\text { status }\end{array}$} & \multicolumn{5}{|c}{ Death due to ND / Total deaths (Proportional mortality due to ND) } \\
\cline { 2 - 6 } & $<9 \mathrm{wks}$ & $9-28 \mathrm{wks}$ & $>28 \mathrm{wks}$ & Male & Female \\
\hline Unvaccinated & $2 / 9$ & $42 / 70^{* *}$ & $8 / 61$ & $15 / 30$ & $37 / 110$ \\
& $(22.2 \%)$ & $(60.0 \%)$ & $(13.1 \%)$ & $(50.0 \%)$ & $(33.6 \%)$ \\
Vaccinated & $6 / 24$ & $4 / 20$ & $0 / 18$ & $3 / 20$ & $7 / 42$ \\
& $(25.0 \%)$ & $(20.0 \%)$ & $(0 \%)$ & $(15.0 \%)$ & $(16.7 \%)$ \\
\hline
\end{tabular}

** Significantly higher $(\mathrm{P}<0.01)$ as compared to two other age group

Isolation and characterization of ND virus

Eight ND-suspected samples were examined for the presence of NDV by embryo inoculation. All the 8 samples caused embryo mortality with HA activity in the allantoic fluid, which could be inhibited by NDV-specific antiserum. Two isolates, one from each village, were taken for further characterization. The mean 
death time (MDT) of inoculated chicken embryos was estimated to be less than 60 hours, which indicated that these isolates were of velogenic pathotype. Velogenic viscerotropic NDV is considered endemic in Bangladesh. Chowdhury et al. (1982) examined 150 NDV isolates obtained from 19 districts of Bangladesh and found 120 (80\%) velogenic by MDT.

In conclusion, vaccination against ND could significantly improve backyard poultry production in Bangladesh. Because of the small flock size it would be impractical to expect that the farmers would vaccinate their backyard chickens on their own. Instead, vaccination coverage needs to be ensured through either the Department of Livestock Services or self-employed village vaccinators.

\section{References}

Agbede G, Demey F, Verhulst A and Bell JG 1992: Prevalence of Newcastle disease in traditional breeding facilities for chickens in Cameroon. Revue Scientifique et Technique $\mathbf{1 1}$ 805-811.

Aini I 1990: Indigenous chicken production in South-East Asia. World's Poultry Science Journal 46 51-57.

Alexander DJ 2001: Gordon Memorial Lecture-Newcastle disease. British Poultry Science 42 $5-22$.

Anon 1996: Newcastle disease. In: Manual of Standards for Diagnostic Tests and Vaccines, 3rd Edn. Office International des Epizooties, Paris. pp. 161-169.

Bell JG and Moulodi S 1988: A reservoir of virulent Newcastle disease virus in village chicken flocks. Preventive Veterinary Medicine 6 37-42.

Chowdhury TIMFR, Sarker AJ, Amin MM and Hossain WIMA 1982: Studies on Newcastle disease in Bangladesh - A Research Report. Department of Microbiology \& Hygiene, Bangladesh Agricultural University, Mymensingh, Bangladesh.

Ezeokoli CD, Umoh JU, Adesiyun AA and Abu P 1984: Prevalence of Newcastle disease virus antibodies in local and exotic chicken under different management systems in Nigeria. Bulletin of Animal Health and Production in Africa 32 253-257.

Harrison JL and Alders RG 2010: An assessment of chicken husbandry including Newcastle disease control in rural areas of Chibuto, Mozambique. Tropical Animal Health and Production 42 729-736.

Hitchner SB, Dommermuth CH, Purchase HG and William JE 1975: Isolation and Identification of Avian Pathogen, American Association of Avian Pathologists, New York.

Otim MO, Kabagambe EK, Mukiibi GM, Christensen H and Bisgaard M 2007: A study of risk factors associated with Newcastle disease epidemics in village free-range chickens in Uganda. Tropical Animal Health and Production 39 27-35.

Schmidt U and Schmidt D 1955: Connection between haemagglutination-inhibition antibodies and immunity after vaccination against Newcastle disease. Archives of Experimental Veterinary Medicine 9 505-516

Thitisak W, Janviriyasopak O, Morries RS, Von Kruedender R and Srihakim S 1989: A poultry health and productivity profile - disease and control measures. In: Proceedings of the International Seminar on Animal Health and Production Services for Village Livestock, Khon Kaen, Thailand, 2-9 August 1989, pp. 409-415. 